\title{
Protective effects of biochanin A on articular cartilage: in vitro and in vivo studies
}

Ding-Qian Wu $\mathrm{W}^{1,2}$, Hui-ming Zhong ${ }^{1}$, Qian-hai Ding ${ }^{3}$ and Li Ba ${ }^{1,2^{*}}$

\begin{abstract}
Background: Increased production of matrix metalloproteinases (MMPS) is closely related to the progression of osteoarthritis $(\mathrm{OA})$. The present study was performed to investigate the potential value of biochanin $\mathrm{A}$ in inhibition of MMP expression in both rabbit chondrocytes and an animal model of OA.

Methods: MTT assay was performed to assess chondrocyte survival in monolayers. The mRNA and protein expression of MMPs (including MMP-1, MMP-3, and MMP-13) and tissue inhibitor of metalloproteinase-1 (TIMP-1) in interleukin-1 < beta $>(\mathrm{IL}-1 \beta)$-induced rabbit chondrocytes were determined by quantitative real-time PCR and enzyme-linked immunosorbent assay (ELISA), respectively. The involvement of the NF-kappaB (NF-kB) pathway activated by IL-1 $\beta$ was determined by western blotting. The in vivo effects of biochanin A were evaluated by intra-articular injection in an experimental OA rabbit model induced by anterior cruciate ligament transection (ACLT).
\end{abstract}

Results: Biochanin A downregulated the expression of MMPs and upregulated TIMP-1 at both the mRNA and protein levels in IL-1 $\beta$-induced chondrocytes in a dose-dependent manner. In addition, IL-1 $\beta$-induced activation of NF-KB was attenuated by biochanin A, as determined by western blotting. Moreover, biochanin A decreased cartilage degradation as determined by both morphological and histological analyses in vivo.

Conclusions: Taken together, these findings suggest that biochanin A may be a useful agent in the treatment and prevention of OA.

Keywords: Biochanin A, Osteoarthritis, Chondroprotection, Interleukin-1 beta, Matrix metalloproteinases, Anterior cruciate ligament transection (ACLT)

\section{Background}

Osteoarthritis $(\mathrm{OA})$ is a multifactorial degenerative joint disorder characterized by the progressive breakdown of cartilage extracellular matrix. OA affects approximately $12 \%$ of the aging population in western countries, while a quarter of people aged over 55 have an episode of persistent knee pain [1]. There is still no effective treatment to block the progression of OA. Other than surgical therapies, treatment for OA has generally been aimed at alleviating the major complaints, such as swelling, pain, and muscle tightness.

\footnotetext{
* Correspondence: liba2013sky@163.com

'Department of Emergency Medicine, The Second Affiliated Hospital of School of Medicine, Zhejiang University, Jie Fang Road 88\#, Hangzhou 310009, People's Republic of China

${ }^{2}$ Research Institute of Emergency Medicine, Jie Fang Road 88\#, Hangzhou 310009, People's Republic of China

Full list of author information is available at the end of the article
}

Homeostasis of the cartilage is maintained by the balance between the anabolic and catabolic activities of the articular chondrocytes [2]. The aggregating proteoglycan along with type II collagen provides robust mechanical properties to cartilage in a healthy joint. Increased type II collagen breakdown by collagenases and aggrecan cleavage by aggrecanases ultimately lead to joint cartilage destruction and exposure of the underlying bone [3]. The matrix metalloproteinases (MMPs) are thought to be the key matrix degradation enzymes due to their ability to cleave most components of the extracellular matrix [4]. In addition, the imbalance between MMPs and tissue inhibitors of metalloproteinases (TIMPs) plays a pathophysiological role in the progression of OA [5]. Inflammatory cytokines, such as interleukin-1 beta (IL$1 \beta$ ), tumor necrosis factor-alpha (TNF- $\alpha$ ), and IL-6, have been detected in the synovial fluid of OA patients [6]. 
For example, IL-1 $\beta$ has been reported to contribute to OA progression by inducing MMP expression as well as other catabolic factors [7].

Currently, there is growing interest in compounds extracted from plants that possess significant value in the treatment of OA for their potent antiarthritic effects and minimal side effects. Biochanin A, an isoflavone found in red clover, has been shown to possess anticancer, antiallergic, and anti-inflammatory effects [8]. A recent study indicated that biochanin A exerted antiproliferative and anti-inflammatory effects through the inhibition of iNOS expression, p38-MAPK and ATF-2 phosphorylation, and blocking of NF- $\mathrm{kB}$ nuclear translocation [9]. A previous study indicated that biochanin A inhibited tumor invasion in human glioblastoma (U87MG) cells by suppressing the enzymatic activities of MMP-2 and MMP-9 [10]. As MMPs are regarded as major factors in the pathophysiology of OA, this close link between biochanin A and MMPs prompted us to explore whether biochanin A could have a protective effect in OA by regulating MMPs. The present study was performed to determine the effects of biochanin A treatment on osteoarthritis in vitro and in vivo.

\section{Methods \\ Reagents}

Biochanin A and 3-(4, 5-Dimethyl-2-thiazolyl)-2, 5diphenyl-2H-tetrazolium bromide (MTT) were obtained from Sigma-Aldrich (St Louis, MO, USA). Recombinatnt interleukin-1beta was purchased from Peprotech Group (USA). Dul-becco's Modified Eagle's Medium (DMEM), penicillin and streptomycin, fetal bovine serum (FBS), 0.05\% trypsin and collagenase II were obtained from GibcoBRL (Grand Island, NY, USA). Biochanin A was dissolved in dimethyl sulphoxide (DMSO) and filtered prior to use.

\section{Isolation and culture of chondrocytes}

The study was approved by the Institutional Animal Care and Use Committee of Zhejiang University (Hangzhou, China) and conducted in accordance with the Guide for the Care and Use of Laboratory Animals as adopted and promulgated by the United States $\mathrm{Na}$ tional Institutes of Health. Normal articular cartilage was collected from the tibial plateau and femoral condyle of 4-week-old New Zealand rabbits. Cartilage was cut into thin slices and digested with $0.4 \%$ collagenase II in DMEM at $37^{\circ} \mathrm{C}$ for $4 \mathrm{~h}$. The released cells were centrifuged, resuspended, and cultured in $75-\mathrm{cm}^{2}$ culture flasks in complete DMEM supplemented with 10\% FBS and antibiotics (100 units/mL penicillin, $100 \mu \mathrm{g} / \mathrm{mL}$ streptomycin). Confluent primary chondrocytes were passaged at a ratio of 1:3. Chondrocytes from passages two to three were used for our experiments.

\section{Assessment of cell viability}

The cytotoxicity of biochanin A to rabbit chondrocytes was evaluated in the presence of increasing concentrations of biochanin A $(0,5,25,50 \mu \mathrm{M})$ by using the MTT assay according to the instructions of the manufacturer as previously described [11]. Briefly, rabbit chondrocytes were seeded onto 96-well plates at a density of $5 \times 10^{3} /$ well. Various concentrations of biochanin A $(5,25,50 \mu \mathrm{M})$ was added to cultured wells and incubated for $24 \mathrm{~h}$. At the indicated time, $20 \mu \mathrm{L}$ of MTT solution $(5 \mathrm{mg} / \mathrm{ml}$ in phosphate-buffered saline) was added to each well and incubated for another $4 \mathrm{~h}$ at $37^{\circ} \mathrm{C}$. After removement of supernatant, $150 \mu \mathrm{L}$ of DMSO was added to each well and absorbance at $570 \mathrm{~nm}$ was measured using a microplate reader (Bio-Rad, Hercules, CA, USA).

\section{Quantitative real-time polymerase chain reaction (PCR)}

Serum-starved chondrocytes were pretreated with various concentrations of biochanin A for $2 \mathrm{~h}$ followed by coincubation with IL-1 $\beta$ for $24 \mathrm{~h}$. At the indicated time, the monolayer chondrocytes were harvested for extraction of total RNA by using Trizol Reagent (Invitrogen, Carlsbad, $\mathrm{CA}$ ), while the culture supernatant was collected for storing at $-80^{\circ} \mathrm{C}$ until use. First complementary DNA (cDNA) was synthesized by using a primescript $-\mathrm{RT}$ reagent kit (TaKaRa Biotechnology Co, Ltd, Japan). Quantitative real-time PCR was performed in $20 \mu \mathrm{L}$ reactions by the following procedures: followed by 40 cycles of $95^{\circ} \mathrm{C}$ for 15 seconds and $60^{\circ} \mathrm{C}$ for 32 seconds. The following primers were used: for MMP-1, 5'-atgctgaaacctgaagatgat-3' (forward) and 5' - ccttggagactttggtgaatgt-3' (reverse); for MMP-3, 5' - cgttcctgatgttggtcactt -3' (forward) and 5' - tcagcctctccttcatacttcc $-3^{\prime}$ (reverse); for MMP-13, 5'-ccctcctcaacagtaacgag-3' (forward) and 5' - agtttgcctgtcacctctaagc-3' (reverse); for TIMP-1, 5'-gggctccagaagtcaatcatac-3' (forward) and 5'-tacccgcagacactttccat-3' (reverse); for 18S, 5'-cgtagttccgaccata aacgat-3' (forward), 5' - aatctgtcaatcctgtccgtgt-3' (reverse). Expression of $18 \mathrm{~S}$ was used as endogenous control. Amplification and quantification of relative expression levels of MMP-1, MMP-3, MMP-13 and TIMP-1 was determined by $\Delta \Delta \mathrm{CT}$ method [12].

\section{ELISA}

The effect of IL-1 $\beta$ and/or biochanin A on the level of MMP-1, MMP-3, MMP-13 and TIMP-1 secreted by rabbit chondrocytes in the culture supernatant mentioned above was furthered detected by using commercially available ELISA kit (R\&D Systems, Inc., Minneapolis, MN, USA) and all the assays were performed in accordance with the manufacturer's instructions. 


\section{Western blot analysis}

Confluent rabbit chondrocytes were starved of serum overnight and pretreated with various concentrations of biochanin A for $2 \mathrm{~h}$, followed by coincubation with IL$1 \beta$ for $24 \mathrm{~h}$. Cells were washed with ice-cold PBS and lysed with lysis buffer. The protein samples were separated by $12 \%$ sodium dodecyl sulfate-polyacrylamide gel electrophoresis (SDS-PAGE) and transferred onto PVDF membranes. The membranes were blocked with $5 \%$ non-fat milk dissolved in TBST buffer and probed with primary antibodies against MMP-1, MMP-3, MMP-13, TIMP-1 (Proteintech, Chicago, IL), IкB- $\alpha$, NF-кB p65, and $\beta$-actin (Cell Signaling Technology, Danvers, MA) at $4^{\circ} \mathrm{C}$ overnight. The membranes were washed with TBST and incubated with appropriate HRP-labeled secondary antibodies for $1 \mathrm{~h}$ at room temperature. Detection was carried out using enhanced chemiluminescent (ECL) substrate and exposure to Kodak X-Omat film (Kodak, Rochester, $\mathrm{NY}$ ) according to the manufacturer's protocols.

\section{Animal studies}

Rabbits were obtained from the Animal Center of Zhejiang University and the procedures were approved by the Institutional Animal Care and Use Committee of Zhejiang University, Hangzhou, China. Twelve mature New Zealand rabbits underwent bilateral anterior cruciate ligament transection (ACLT) and divided randomly into the lowconcentration (A) and high-concentration (B) biochanin A treatment groups. Four weeks after ACLT surgery, rabbits in groups $\mathrm{A}$ and $\mathrm{B}$ received an intra-articular injection of $0.3 \mathrm{~mL}$ of 5 and $25 \mu \mathrm{M}$ biochanin A, respectively, into the left knee and $0.3 \mathrm{~mL}$ of DMSO (vehicle) into the right knee. Weekly administration continued for 5 weeks. All rabbits were sacrificed after 9 weeks of ACLT surgery. Four rabbits were used as a normal control group.

\section{Histological assessment}

Collected specimens of femoral condyle were fixed in $10 \%$ neutral-buffered formalin for $72 \mathrm{~h}$. After dehydration through an alcohol series, specimens were embedded in paraffin, cut into sections $7 \mu \mathrm{m}$ thick, and stained with Safranin-O-fast green. The degree of cartilage degradation was scored according to the Mankin system by two independent researchers in a blinded manner [13].

\section{Evaluation of cartilage gene expression by quantitative real-time PCR}

Collected cartilage was frozen in liquid nitrogen prior to use. The mRNA levels of MMP-1, MMP-3, MMP-13, and TIMP-1 in all cartilage samples were assessed by quantitative real-time PCR as described above.

\section{Statistical analysis}

All experiments were performed duplicated and repeated three times using independent samples. The results are expressed as means \pm standard deviation (SD). One-way ANOVA followed by Dunnett's analysis was used for statistical analysis. In all analyses, $P<0.05$ was taken to indicate statistical significance.

\section{Results}

Effects of biochanin A on chondrocytes viability

MTT assay was performed to evaluate the cytotoxicity of biochanin A on rabbit chondrocytes. As shown in Figure 1, biochanin A at 5 to $50 \mu \mathrm{M}$ exhibited no significant cytotoxicity toward rabbit chondrocytes.

\section{Effects of biochanin A on gene expression of MMP-1,} $-3,-13$, and TIMP-1 induced by IL- $1 \beta$ in chondrocytes Quantitative real-time PCR was performed to assess the mRNA levels of MMP-1, $-3,-13$, and TIMP-1 in chondrocyte monocultures. As shown in Figure 2, IL-1 $\beta$ enhanced the expression of MMP-1, -3 , and -13 , and decreased the expression of TIMP-1. In chondrocytes pretreated with biochanin A, the upregulated MMP-1, -3, and -13 expression was markedly inhibited and the downregulated TIMP-1 expression was enhanced.

\section{Effects of biochanin A on protein expression of MMP-1,} $-3,-13$, and TIMP-1

To further investigate MMP and TIMP-1 protein levels in IL-1 $\beta$-stimulated chondrocytes, we used enzyme-linked immunosorbent assay (ELISA) and western blotting. As shown in Figures 3, 4 and 5, MMP-1, -3 , and -13 protein levels were increased after IL-1 $\beta$ stimulation, and this effect was markedly suppressed by biochanin A in a dosedependent manner. Although $5 \mu \mathrm{M}$ biochanin A showed no significant effects, a higher concentration of biochanin A markedly suppressed the production of MMP-1, -3,

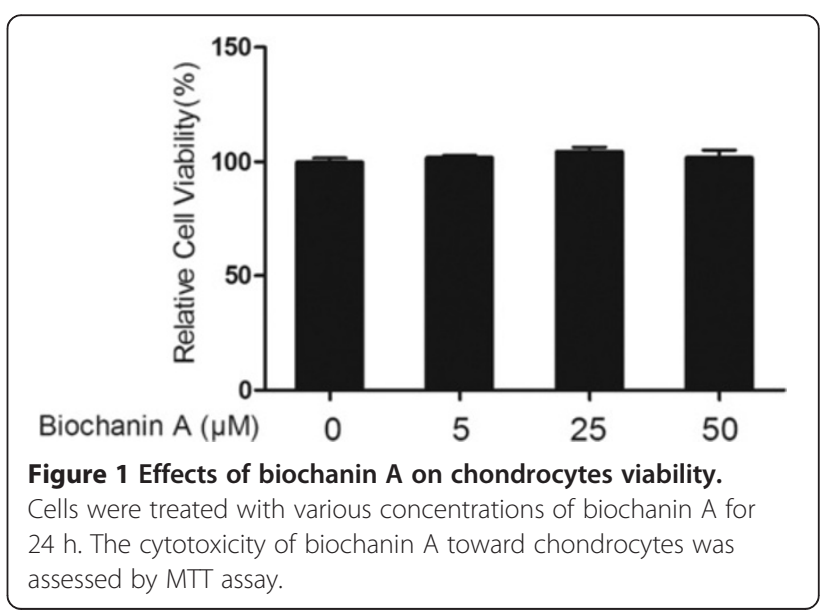


(a)

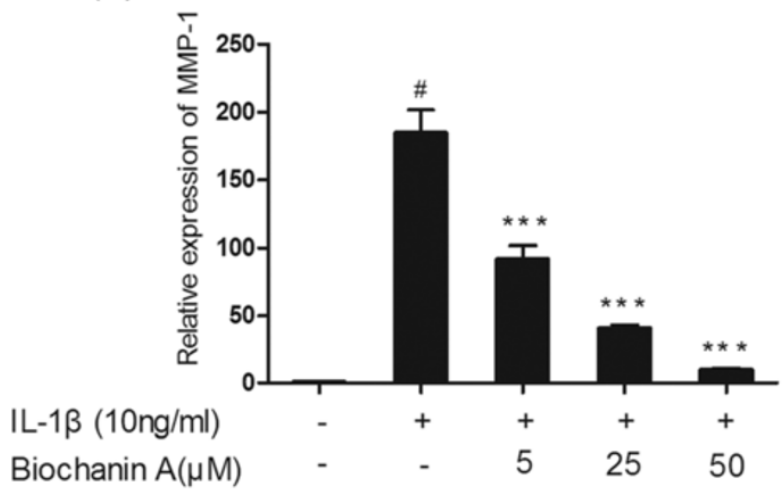

(c)

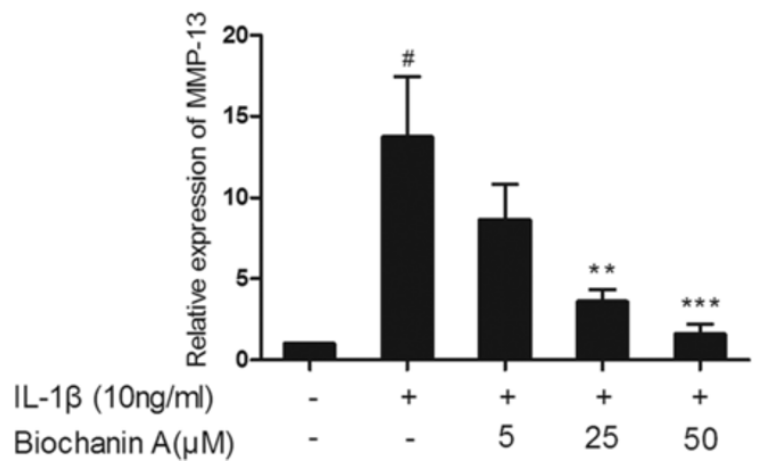

(b)

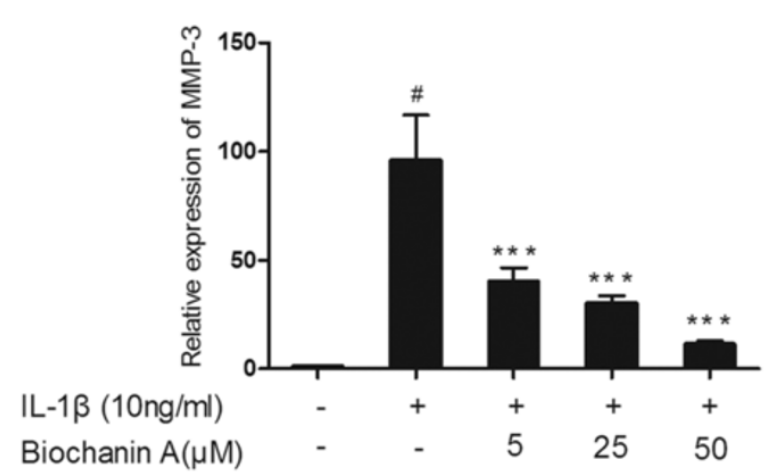

(d)

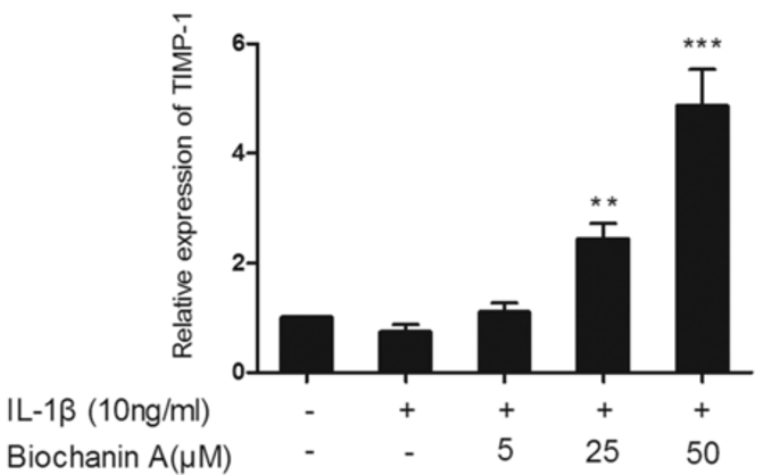

Figure 2 Relative gene expression of MMP-1, $-3,-13$ and TIMP-1 in IL-1 $\beta$-induced chondrocytes. Cells were pre-treated with different concentrations of biochanin A for $2 \mathrm{~h}$, followed by co-incubation with IL-1 $\beta$ (10 ng/ml) for $24 \mathrm{~h}$. Data were expressed as means \pm standard deviation (SD). ${ }^{* P}<0.05$, ${ }^{* * P}<0.01,{ }^{* *} \mathrm{P}<0.001$, compared with cells stimulated with IL-1 $\beta$ alone; \# $\mathrm{P}<0.01$, compared with normal chondrocytes. (a), (b), (c), (d) represented as the relative gene expression of MMP-1,MMP-3,MMP-13 and TIMP-1 respectively.

and -13 and promoted the production of TIMP-1, consistent with the quantitative real-time PCR results.

Effects of biochanin A on IKB-a degradation and NF-KB activation in IL-1 $\beta$-treated chondrocytes

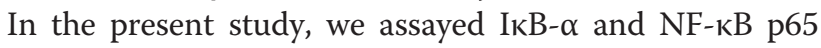
to evaluate the involvement of the NF- $\mathrm{kB}$ pathways activated by IL-1 $\beta$. As expected, biochanin A inhibited IL$1 \beta$-stimulated activation of NF-kB p65 and suppressed the IL-1 $\beta$-induced degradation of $\mathrm{I} \kappa \mathrm{B}-\alpha$, as shown in Figures 6 and 7 .

\section{Gross morphology and histological evaluation}

Our in vivo data revealed severe cartilage erosion in all rabbits treated by ACLT. While rabbits that received intra-articular injection of biochanin A showed less cartilage destruction compared with the vehicle-treated control group, the difference was not significant. On histological examination, cartilage specimens from the vehicle-treated group showed noticeable morphological changes, including surface irregularities, hypocellularity, and reduction of Safranin-O staining. While a significant decrease in the severity of cartilage degradation was seen in biochanin A -treated groups, particularly in the $50 \mu \mathrm{M}$ group (Figure 8), which was consistent with histological analyses using the Mankin scoring system (Table 1).

\section{Effects of biochanin A on MMP-1, $-3,-13$, and TIMP-1 gene expression}

As shown in Figure 9, in vehicle-treated cartilage of OA rabbits, the relative levels of MMP-1, -3 , and -13 mRNA expression were markedly increased, while that of TIMP-1 was decreased compared to the levels in normal cartilage. However, MMP-1, -3 , and -13 mRNA levels were downregulated and that of TIMP-1 was upregulated in the biochanin-A-treated group in comparison with vehicle-treated cartilage.

\section{Discussion}

The results of the present study indicated that biochanin A potently suppressed MMP-1, -3 , and -13 expression 
(a)

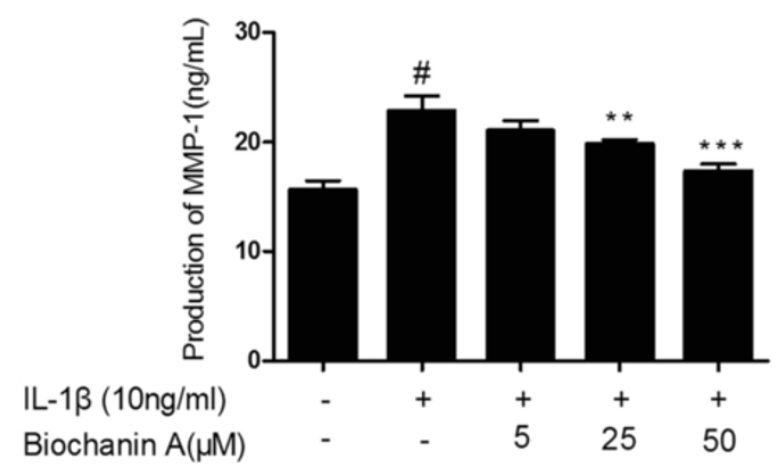

(c)

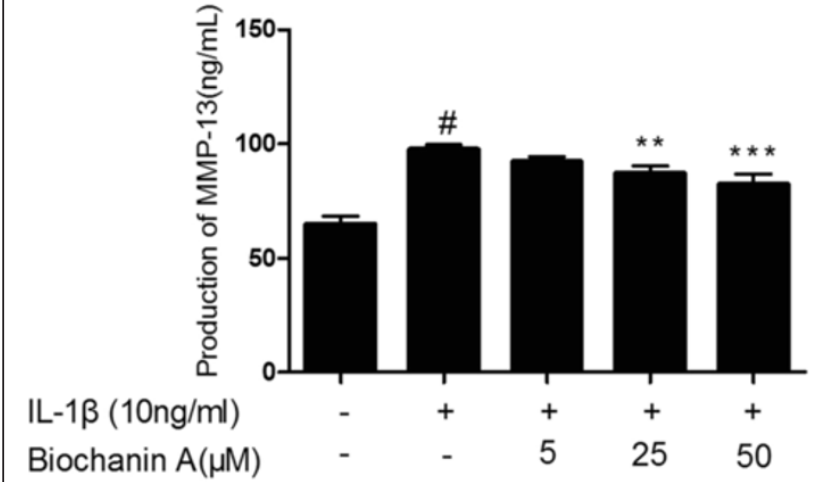

(b)

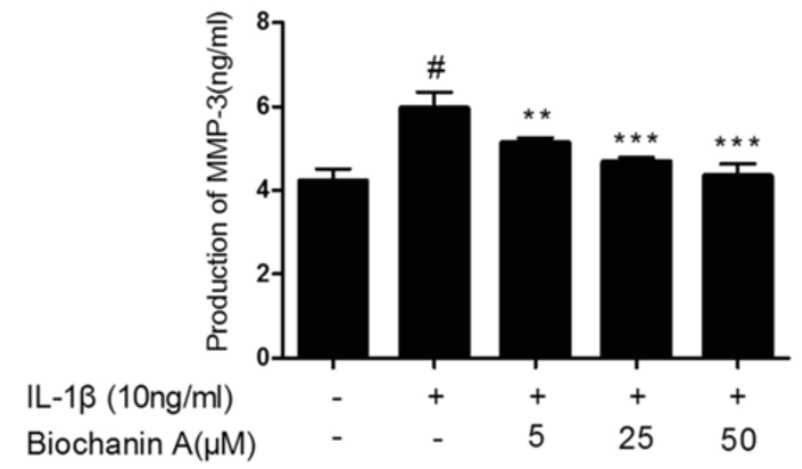

(d)

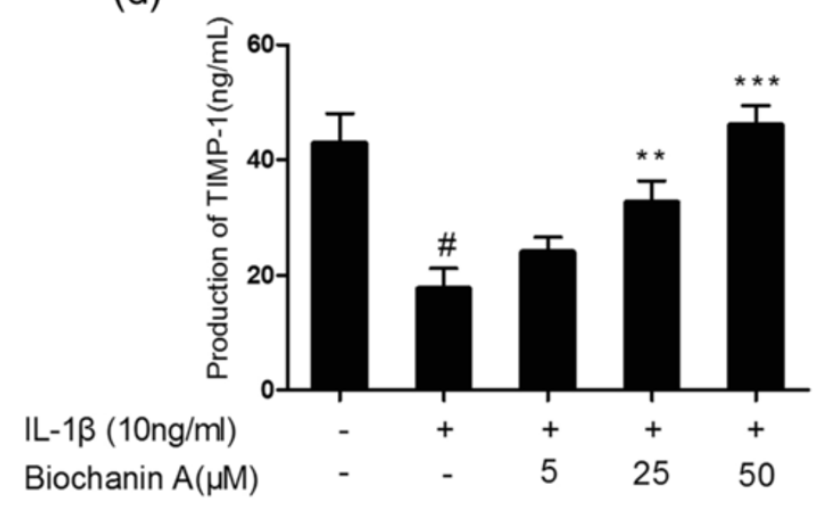

Figure 3 Production of MMP-1, $\mathbf{- 3}, \mathbf{- 1 3}$, and TIMP-1 in cultured supernatant. Production of MMP-1, $-3,-13$, and TIMP-1 was analyzed by enzyme-linked immunosorbent assay (ELISA). Data were expressed as means \pm standard deviation (SD). ${ }^{*} P<0.05,{ }^{* *} P<0.01$, ${ }^{* * *} P<0.001$, compared with cells stimulated with IL-1 $\beta$ alone; \#P <0.01, compared with normal chondrocytes. (a), (b), (c), (d) represented as the production of MMP-1,MMP-3,MMP-13 and TIMP-1 respectively.

and increased that of TIMP-1 both in vitro and in vivo. In addition, our results revealed the involvement of NF$\kappa \mathrm{B}$ in MMP regulation by biochanin $\mathrm{A}$.

Current therapeutic options for OA include Nonsteroidal anti-inflammatory drugs [14] such as cycloo- xygenase-2 inhibitors and other agents for pain relief but these drugs fail to block the progression of the disease. Apart from this, these clinical agents are associated with many risks including gastrointestinal [15], cardiovascular [16] and other adverse events. There is an
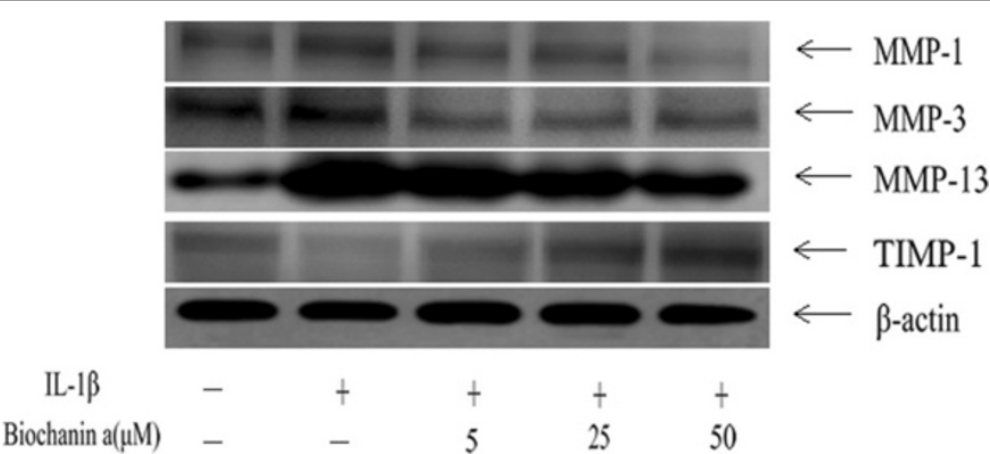

Figure 4 Effects of biochanin A on protein expression of MMP-1,-3, -13, and TIMP-1 in interleukin-1 $\beta$ (IL-1 $\beta$ )-induced chondrocytes. Chondrocytes were incubated for 24 hours with IL-1 $\beta$ or a combination of biochanin A and IL-1 3 . Protein levels of MMP-1, $-3,-13$, and TIMP-1 were determined by Western blotting. $\beta$-actin was used as a loading control in the Western blotting. 


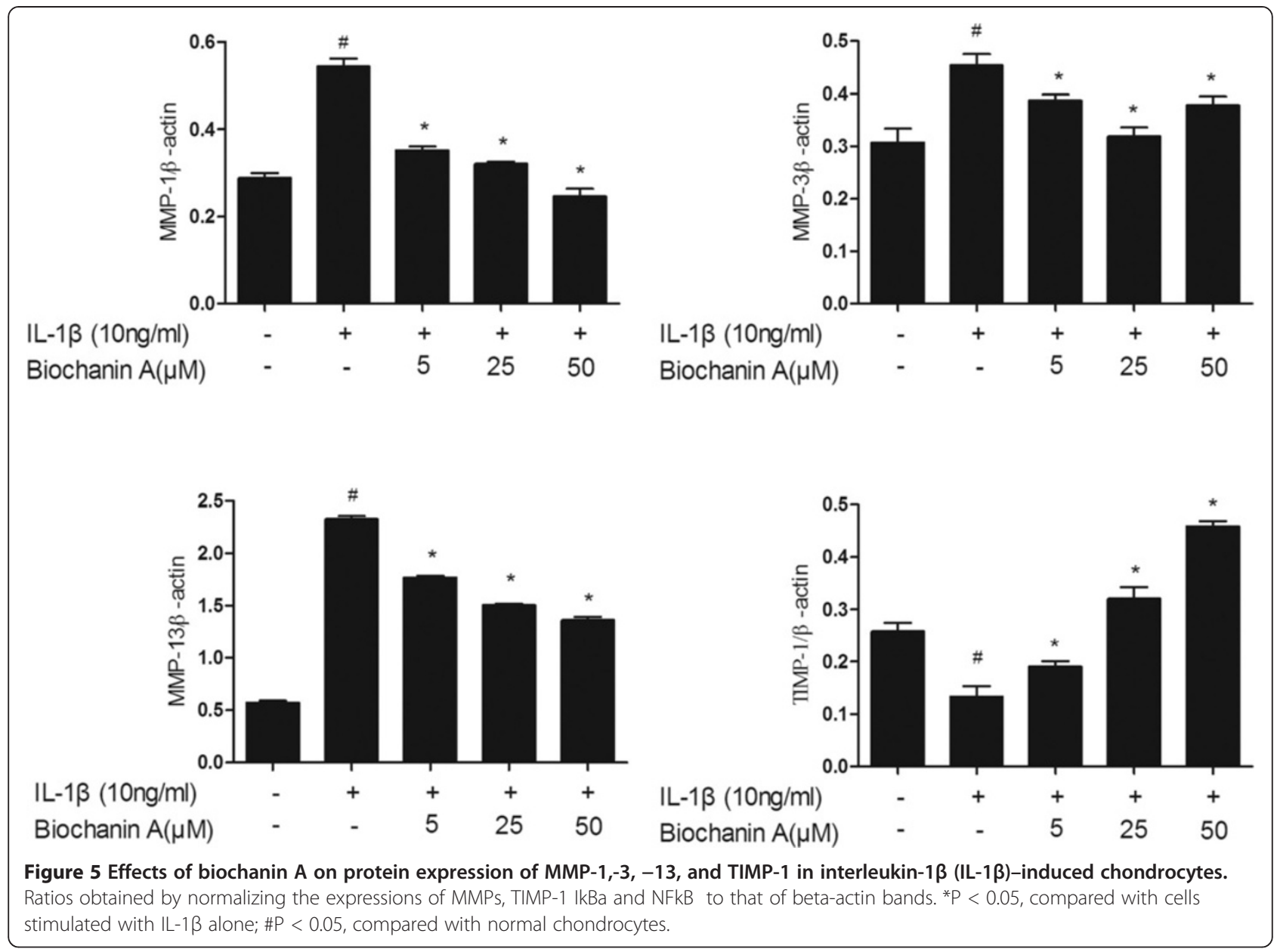

increasing interest in use of compounds derived from natural plants or herbs for the treatment of OA because they have shown to be clinical efficacy with minimal side effects, as compared to routine pharmacological strategies. Biochanin A, an isoflavone derived from red clover, cabbage, and alfalfa, has been reported to exert a wide range of pharmacological effects in different experimental models.
Sehdev et al. reported that biochanin A may be a unique natural anticancer agent that can selectively target cancer cells and inhibit multiple signaling pathways in HER-2positive breast cancer cells [17]. Lee et al. suggested that biochanin A may play important physiological roles in the prevention of postmenopausal osteoporosis in osteoblastic MC3T3-E1 cells [18]. Chen et al. reported that biochanin

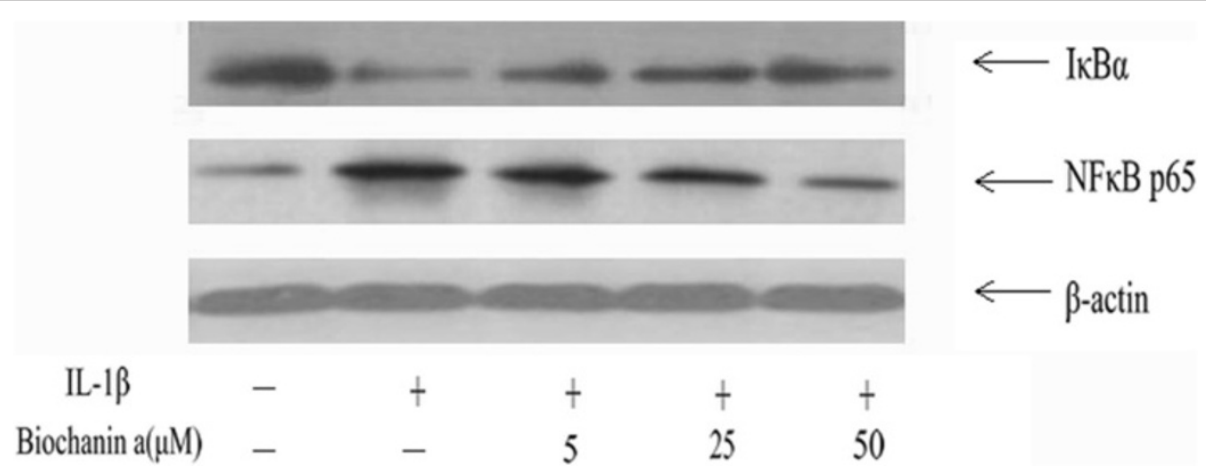

Figure 6 Effects of biochanin A on IKB- $\alpha$ degradation and NF-KB activation in IL-1 $\beta$-induced chondrocytes. Chondrocytes were pre-treated with various concentrations of biochanin A for $2 \mathrm{~h}$, followed by co-incubation with IL-1 $\beta$ ( $10 \mathrm{ng} / \mathrm{ml})$ for $24 \mathrm{~h}$, and harvested for Western blotting analysis. Biochanin A suppressed IL-1 $\beta$-induced degradation of IKB- $\alpha$ and then attenuated IL-1 $\beta$-stimulated activation of NF-K B. 


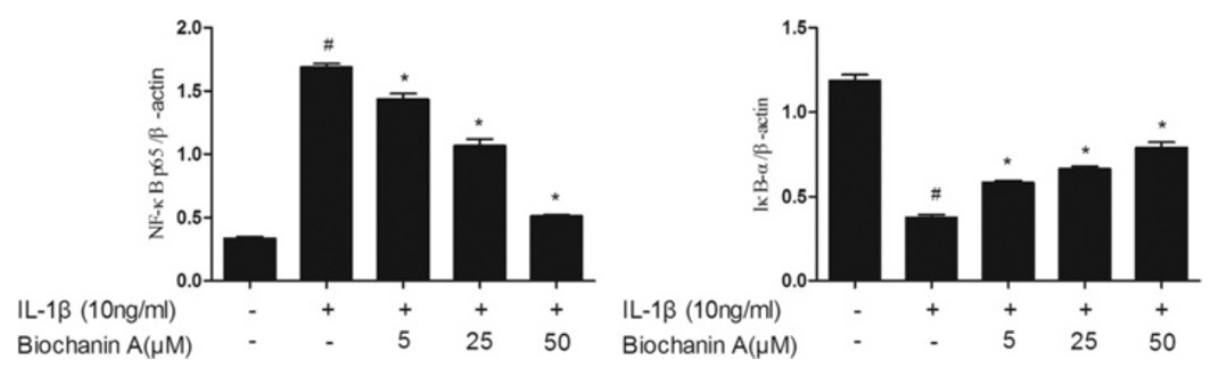

Figure 7 Effects of biochanin A on IKB- $\alpha$ degradation and NF-KB activation in IL-1 $\beta$-induced chondrocytes. Ratios obtained by normalizing the expressions of IKB- $\alpha$ and NF-kB to that of beta-actin bands. ${ }^{*} P<0.05$, compared with cells stimulated with IL-1 $\beta$ alone; $\# P<0.05$, compared with normal chondrocytes. The former and latter image represented as the relative expression of NF-k B p65 and IkB-a respectively.

A protects dopaminergic neurons against LPS-induced damage through inhibition of microglia activation and generation of proinflammatory factors [19]. In addition, biochanin A was reported to inhibit MMP-9 and MT1-MMP in human glioblastoma (U87MG) cells. However, little is known about the effects of biochanin A on osteoarthritic models or chondrocytes. Recent study showed that biochanin A prevents adipogenesis, enhances osteoblast differentiation in mesenchymal stem cells, and has beneficial regulatory effects in bone formation [20]. In view of the uncertain effects of biochanin A on osteoarthritis as well as the close link between biochanin A and MMPs, the present study was performed to explore the potential use of biochanin A in vitro and in vivo.

Osteoarthritis (OA) is characterized by the loss of the balance between anabolic and catabolic responses of stimulated chondrocytes, driven locally by a series of cytokines of which IL-1 $\beta$ is regarded as the major factor [21]. IL-1 $\beta$ is thought to be one of the most important catabolic cytokines produced by chondrocytes in OA [22]. IL-1 $\beta$ is known to be a potent inducer of enzymes, prostanoids, nitric oxide, and free radicals [23]. Moreover, IL-1 $\beta$ can suppress the synthesis and production of type II collagen and proteoglycans [24,25]. Upregulation (a)

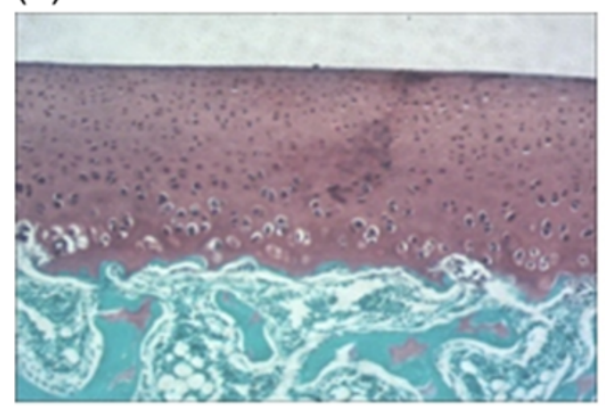

(c)

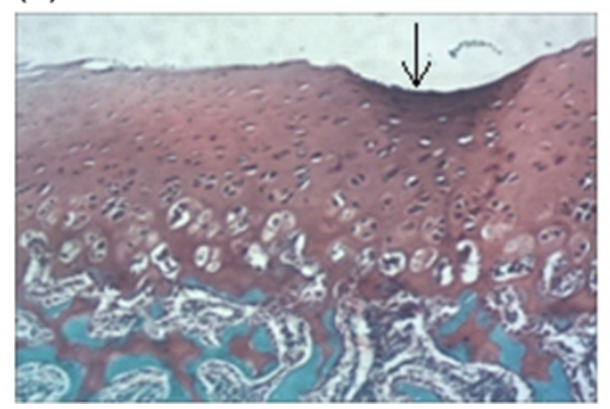

(b)

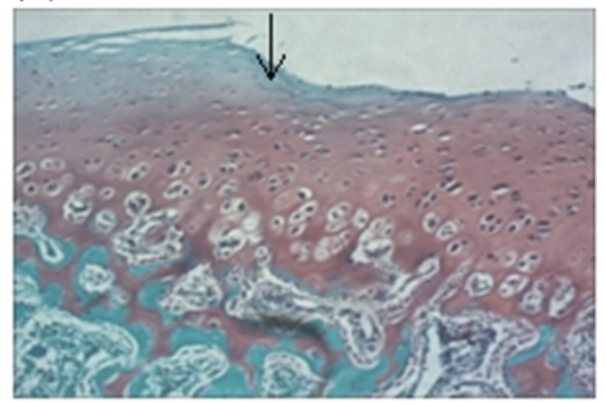

(d)

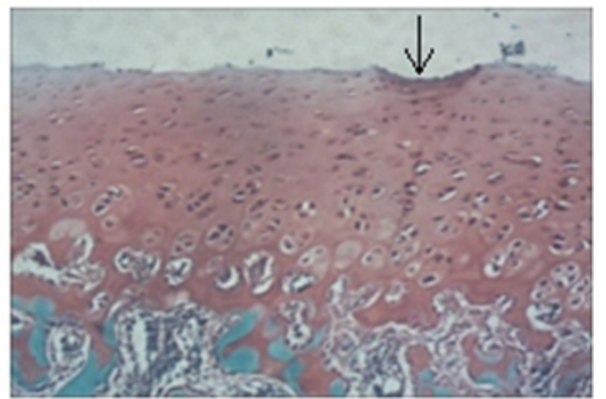

Figure 8 Photographs of rabbit articular cartilage stained by Safranin-O. (a). Normal articular cartilage showed no reduction of Safranin staining. (b). Vehicle-treated cartilage showed the loss of Safranin O staining, the arrow indicates the calcified cartilage and irregular surface. (c), (d) biochanin A -treated cartilage showed reduction loss of Safranin O staining compared with vehicle-treated group (original magnification $\times 40$ ), the arrow indicate irregular surface. $\mathbf{a}, \mathbf{b}, \mathbf{c}, \mathbf{d}$ in Figure 8 represented as normal cartilage, vehicle-treated cartilage, biochanin A (5 $\mu \mathrm{M})$ and biochanin A (50 $\mu \mathrm{M})$-treated cartilage respectively. 
Table 1 Histological score of articular cartilage

\begin{tabular}{llll}
\hline Femoral condyle & Vehicle & $\begin{array}{l}\text { Biochanin A } \\
(\mathbf{5} \boldsymbol{\mu M})\end{array}$ & $\begin{array}{l}\text { Biochanin A } \\
(\mathbf{5 0} \boldsymbol{\mu M})\end{array}$ \\
\hline Structural changes & $3.23 \pm 0.31$ & $2.76 \pm 0.15$ & $1.8 \pm 0.1^{*}$ \\
Cellular changes & $2.67 \pm 0.15$ & $2.27 \pm 0.25$ & $1.87 \pm 0.15^{*}$ \\
Safranin staining & $2.9 \pm 0.1$ & $2.53 \pm 0.21$ & $1.94 \pm 0.15^{*}$ \\
Total score & $8.8 \pm 0.53$ & $7.57 \pm 0.40^{*}$ & $5.6 \pm 0.26^{*}$ \\
\hline
\end{tabular}

Values are the mean \pm SD. ${ }^{*} p<0.05$ compared with vehicle group.

of IL-1 $\beta$ in OA cartilage tissue has been reported; indeed, increased levels of IL- $1 \beta$ have been detected in the synovial fluid of patients with OA [26,27]. Our data confirmed that IL-1 $\beta$ increased the expression levels of MMPs (including MMP-1, -3 , and -13) and decreased the expression of TIMP-1 in chondrocytes, consistent with previous reports $[28,29]$.

MMPs comprise a family of enzymes that facilitate extracellular matrix (ECM) turnover and breakdown. All members of the MMP family have been linked to disease development, notably arthritis, cancer metastasis, chronic inflammation, and neurological disorders [30]. Among all MMPs, MMP-13 has been reported to play a central role in the progression of OA due to its potent proteolytic effects on collagen II, the main component of the ECM. The specialized role of MMP-13 in bone development and disease progression has made it an attractive target for selective MMP-13 inhibitors as therapeutic compounds [31,32]. MMP-1 is a secreted enzyme and has wide substrate specificity for degradation of collagens, aggrecan, versican, serpins, and tenascin- $C$ [33]. The substrate specificity of MMP-3 is also broad; MMP-3 has been shown to break down a number of ECM proteins, including fibronectin, laminin, denatured collagens, and proteoglycans. In addition to ECM degradation, MMP-3 is involved in the activation cascades of MMP-13 and gelatinases. Since MMP-1 and MMP-3 play vital roles in ECM turnover, their regulation has been suggested to be useful in the treatment of OA [34]. Interestingly, our results demonstrated that the upregulated MMP-1, -3 , and -13 expression in IL-1 $\beta$-stimulated chondrocytes were all markedly decreased by biochanin A in a dose-dependent manner. In view of the effects of biochanin A on MMP-2 and MMP-9 as reported previously [10], we also detected the expresson and production of MMP-2 and MMP-9. However, the results were not statistically significant. TIMPs, the endogenous regulators of MMPs, play important roles in maintaining homoeostasis with MMPs. Imbalance between MMPs and TIMPs is a salient feature during $\mathrm{OA}$ progression, leading to disruption of
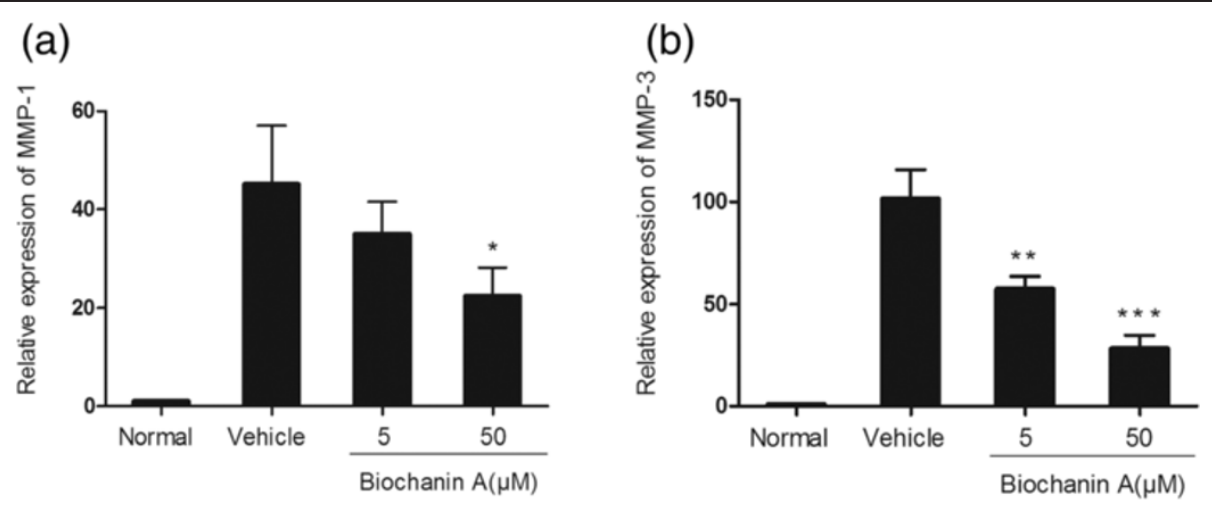

(c)

(d)
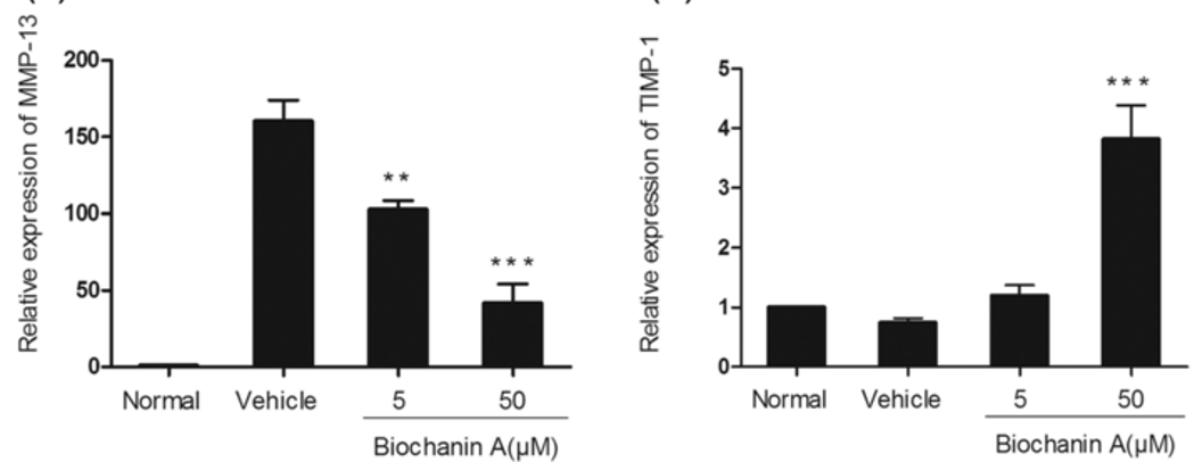

Figure 9 Relative gene expression in articular cartilage. The relative gene expression of MMP-1, $-3,-13$ and TIMP-1 was calculated by using quantitative real-time PCR. Data were presented as means \pm standard deviation (SD). ${ }^{*} P<0.05,{ }^{*} \mathrm{P}<0.01,{ }^{* * *} \mathrm{P}<0.001$, compared with vehicle-treated cartilage. (a), (b), (c), (d) represented as the relative gene expression of MMP-1,MMP-3,MMP-13 and TIMP-1 respectively. 
the balance between ECM biosynthesis and degradation. Since the activities of MMPs can be inhibited by TIMPs, we investigated the effects of biochanin A on TIMP-1 expression; our results suggested upregulation of TIMP-1 by biochanin A in IL-1 $\beta$-stimulated chondrocytes. Hence, we speculated that biochanin A may exert its chondroprotective effects by regulating the expression and activity of MMPs and TIMPs.

The NF- $\mathrm{kB}$ signaling pathway is known to play a vital role in OA $[35,36]$. In the progression of OA, NF- $\mathrm{kB}$ transcriptional factors can be triggered by a number of stimuli, such as cytokines, excessive mechanical stress and degradation products of ECM. Activated NF- $\mathrm{kB}$ regulates several enzymes involved in matrix degradation, including MMP-1, -3 , and -13 [37]. In general, NF- $\kappa B$ p65 is associated with IкB- $\alpha$ in an inactive form in the cytoplasm. Following ІкB- $\alpha$ phosphorylation by stimuli, such as IL-1 $\beta$, the nuclear localization signal is no longer masked, leading to translocation of NF- $\mathrm{KB}$ p 65 to the nucleus [38]. Since biochanin A inhibited the NF- $k B$ activation pathway in other experimental models [9,39], we investigated the involvement of the NF- $\mathrm{KB}$ signaling pathway in MMP modulation by biochanin A in chondrocytes. In this study, we showed that biochanin A inhibited IкB- $\alpha$ degradation and then attenuated the activation of NF- $\mathrm{kB}$ p65. However, further studies of the mechanism underlying the effect of biochanin $\mathrm{A}$ in the treatment of $\mathrm{OA}$ are required.

In addition, we used the ACLT (anterior cruciate ligament transection) model to assess the effects of biochanin A in vivo, which has proven to be effective and reliable $[40,41]$. Pickarski $\mathrm{M}$ et al. reported that matrix degradation and up-regulation of MMPs were obviously observed in the surgically-induced models of osteoarthritis [42]. Similar findings were reported by $\mathrm{Li} \mathrm{X}$ et al., they found that MMP-13 was strikingly up-regulated in ACLT-induced OA [43]. Consistent with our in vitro data, the results of the in vivo study showed that the levels of MMP-1, -3 , and -13 expression were decreased, while that of TIMP-1 was increased, in biochanin-A-treated cartilage compared with vehicle-treated controls. Moreover, we demonstrated that intra-articular injection of biochanin A ameliorated cartilage degradation during the progression of OA. Taken together, these findings confirm the potential value of biochanin $\mathrm{A}$ in the treatment of OA.

\section{Conclusions}

In conclusion, we demonstrated at both the mRNA and protein levels that biochanin A inhibited the expression of MMP-1, -3, and -13 and increased the expression of TIMP-1, all of which are classic biomarkers of inflammation and cartilage degradation in OA. Our study provided strong evidence that these protective effects of biochanin $\mathrm{A}$ are mediated at least in part via inhibition of the NF- $\mathrm{kB}$ pathway. Taken together, these findings indicate that biochanin A exerts anticatabolic effects in the progression of $\mathrm{OA}$ and may reduce the rate of cartilage degradation. Therefore, biochanin A may be useful for the treatment and prevention of OA.

\section{Abbreviations}

MMP: Matrix metalloproteinases; TIMP-1: Tissue inhibitor of metalloproteinases-1; IL-1 B: Interleukin-1 beta; OA: Osteoarthritis; NF-KB: Natural factor kappa B; IKB-a: Inhibitory kappa B-alpha; ACLT: Anterior cruciate ligament transection.

\section{Competing interests}

The authors declare that they have no competing interests.

\section{Authors' contributions}

All authors have read and approved the final manuscript. D-OW, H-MZ and Q-HD performed the experiments, analyzed the data, prepared the figures and wrote the manuscript. LB interpreted the data and edited the manuscript.

\section{Acknowledgements}

This study was supported by a grant from the National Natural Science Foundation of China (81201429).

\section{Author details}

Department of Emergency Medicine, The Second Affiliated Hospital of School of Medicine, Zhejiang University, Jie Fang Road 88\#, Hangzhou 310009, People's Republic of China. ${ }^{2}$ Research Institute of Emergency Medicine, Jie Fang Road 88\#, Hangzhou 310009, People's Republic of China. ${ }^{3}$ Department of Orthopedic Surgery, The Second Affiliated Hospital of School of Medicine, Zhejiang University, Jie Fang Road 88\#, Hangzhou 310009, People's Republic of China.

Received: 23 April 2014 Accepted: 29 October 2014

Published: 15 November 2014

\section{References}

1. Hunter DJ, Felson DT: Osteoarthritis. BMJ 2006, 332:639-642.

2. Goldring MB, Marcu KB: Cartilage homeostasis in health and rheumatic diseases. Arthritis Res Ther 2009, 11(3):224.

3. Struglics A, Larsson S, Pratta MA, Kumar S, Lark MW, Lohmander LS: Human osteoarthritis synovial fluid and joint cartilage contain both aggrecanase- and matrix metalloproteinase-generated aggrecan fragments. Osteoarthritis Cartilage 2006, 14:101-113.

4. Burrage PS, Mix KS, Brinckerhoff CE: Matrix metalloproteinases: role in arthritis. Front Biosci 2006, 11:529-543.

5. Woessner JF Jr: MMPs and TIMPs-an historical perspective. Mol Biotechnol 2002, 22:33-49.

6. Goldring SR, Goldring MB: The role of cytokines in cartilage matrix degeneration in osteoarthritis. Clin Orthop Relat Res 2004, (427 Suppl):S27-S36.

7. Kobayashi M, Squires GR, Mousa A, Tanzer M, Zukor DJ, Antoniou J, Feige U, Poole AR: Role of interleukin-1 and tumor necrosis factor alpha in matrix degradation of human osteoarthritic cartilage. Arthritis Rheum 2005, 52:128-135.

8. Ko WC, Lin LH, Shen HY, Lai CY, Chen CM, Shih CH: Biochanin a, a phytoestrogenic isoflavone with selective inhibition of phosphodiesterase 4, suppresses ovalbumin-induced airway hyperresponsiveness. Evid Based Complement Alternat Med 2011, 2011:635058.

9. Kole L, Giri B, Manna SK, Pal B, Ghosh S: Biochanin-A, an isoflavon, showed anti-proliferative and anti-inflammatory activities through the inhibition of iNOS expression, p38-MAPK and ATF-2 phosphorylation and blocking NFkappaB nuclear translocation. Eur J Pharmacol 2011, 653:8-15.

10. Puli S, Lai JC, Bhushan A: Inhibition of matrix degrading enzymes and invasion in human glioblastoma (U87MG) cells by isoflavones. J Neurooncol 2006, 79:135-142. 
11. Syed HM, Green L, Bianski B, Jobe CM, Wongworawat MD: Bupivacaine and triamcinolone may be toxic to human chondrocytes: a pilot study. Clin Orthop Relat Res 2011, 469:2941-2947.

12. Pfaffl MW: A new mathematical model for relative quantification in real-time RT-PCR. Nucleic Acids Res 2001, 29:e45.

13. Mankin HJ, Dorfman H, Lippiello L, Zarins A: Biochemical and metabolic abnormalities in articular cartilage from osteo-arthritic human hips. II. Correlation of morphology with biochemical and metabolic data. J Bone Joint Surg Am 1971, 53:523-537.

14. Altman RD: Practical considerations for the pharmacologic management of osteoarthritis. Am J Manag Care 2009, 15:S236-S243.

15. Chan FK, Cryer B, Goldstein JL, Lanas A, Peura DA, Scheiman JM, Simon LS, Singh G, Stillman MJ, Wilcox CM, Berger MF, Breazna A, Dodge W: A novel composite endpoint to evaluate the gastrointestinal (GI) effects of nonsteroidal antiinflammatory drugs through the entire GI tract. J Rheumatol 2010, 37:167-174.

16. Hsiao FY, Tsai YW, Huang WF: Changes in physicians' practice of prescribing cyclooxygenase-2 inhibitor after market withdrawal of rofecoxib: a retrospective study of physician-patient pairs in Taiwan. Clin Ther 2009, 31:2618-2627.

17. Sehdev V, Lai JC, Bhushan A: Biochanin a modulates cell viability, invasion, and growth promoting signaling pathways in HER-2-positive breast cancer cells. J Oncol 2009, 2009:121458.

18. Lee $\mathrm{KH}$, Choi EM: Biochanin A stimulates osteoblastic differentiation and inhibits hydrogen peroxide-induced production of inflammatory mediators in MC3T3-E1 cells. Biol Pharm Bull 2005, 28:1948-1953.

19. Chen $H Q$, Jin $Z Y$, Li GH: Biochanin A protects dopaminergic neurons against lipopolysaccharide-induced damage through inhibition of microglia activation and proinflammatory factors generation. Neurosci Lett 2007, 417:112-117.

20. Su SJ, Yeh YT, Su SH, Chang KL, Shyu HW, Chen KM, Yeh H: Biochanin a promotes osteogenic but inhibits adipogenic differentiation: evidence with primary adipose-derived stem cells. Evid Base Compl Alternative Med. eCAM 2013, 2013:846039.

21. Tchetina EV: Developmental mechanisms in articular cartilage degradation in osteoarthritis. Arthritis 2011, 2011:683970.

22. Goldring MB: Osteoarthritis and cartilage: the role of cytokines. Curr Rheumatol Rep 2000, 2:459-465.

23. Goldring MB, Otero M, Tsuchimochi K, ljiri K, Li Y: Defining the roles of inflammatory and anabolic cytokines in cartilage metabolism. Ann Rheum Dis 2008, 67(Suppl 3):iii75-iii82.

24. Pelletier JP, Martel-Pelletier J, Abramson SB: Osteoarthritis, an inflammatory disease: potential implication for the selection of new therapeutic targets. Arthritis Rheum 2001, 44:1237-1247.

25. Chevalier X: Upregulation of enzymatic activity by interleukin-1 in osteoarthritis. Biomed Pharmacother 1997, 51:58-62.

26. Tetlow LC, Adlam DJ, Woolley DE: Matrix metalloproteinase and proinflammatory cytokine production by chondrocytes of human osteoarthritic cartilage: associations with degenerative changes. Arthritis Rheum 2001, 44:585-594.

27. Westacott Cl, Sharif M: Cytokines in osteoarthritis: mediators or markers of joint destruction? Semin Arthritis Rheum 1996, 25:254-272.

28. Huang BD, He AS, Fu M, Sheng PY, Liao WM: Sinomenine suppresses expression of interleukin-1 beta-induced matrix metalloproteinases in human osteoarthritic chondrocytes. J Med Plant Res 2010, 4:1830-1836.

29. Toegel S, Wu SQQ, Otero M, Goldring MB, Leelapornpisid P, Chiari C, Kolb A, Unger FM, Windhager $\mathrm{R}$, Viernstein $\mathrm{H}$ : Caesalpinia sappan extract inhibits IL1 beta-mediated overexpression of matrix metalloproteinases in human chondrocytes. Genes Nutr 2012, 7:307-318.

30. Klein T, Bischoff R: Physiology and pathophysiology of matrix metalloproteases. Amino Acids 2011, 41:271-290.

31. Heim-Riether A, Taylor SJ, Liang S, Gao DA, Xiong Z, Michael August E, Collins BK, Farmer BT 2nd, Haverty K, Hill-Drzewi M, Junker HD, Mariana Margarit S, Moss N, Neumann T, Proudfoot JR, Keenan LS, Sekul R, Zhang Q, Li J, Farrow NA: Improving potency and selectivity of a new class of non-Znchelating MMP-13 inhibitors. Bioorg Med Chem Lett 2009, 19:5321-5324.

32. Hu Y, Xiang JS, DiGrandi MJ, Du X, Ipek M, Laakso LM, Li J, Li W, Rush TS, Schmid J, Skotnicki JS, Tam S, Thomason JR, Wang Q, Levin Jl: Potent, selective, and orally bioavailable matrix metalloproteinase-13 inhibitors for the treatment of osteoarthritis. Bioorg Med Chem 2005, 13:6629-6644.
33. McCawley $\sqcup$, Matrisian LM: Matrix metalloproteinases: they're not just for matrix anymore! Curr Opin Cell Biol 2001, 13:534-540.

34. Jo H, Park JS, Kim EM, Jung MY, Lee SH, Seong SC, Park SC, Kim HJ, Lee MC: The in vitro effects of dehydroepiandrosterone on human osteoarthritic chondrocytes. Osteoarthritis Cartilage / OARS Osteoarthritis Res Soc 2003, 11:585-594.

35. Roman-Blas JA, Jimenez SA: NF-kappaB as a potential therapeutic target in osteoarthritis and rheumatoid arthritis. Osteoarthritis Cartilage 2006, 14:839-848

36. Lauder SN, Carty SM, Carpenter CE, Hill RJ, Talamas F, Bondeson J, Brennan $P$, Williams AS: Interleukin-1 beta induced activation of nuclear factor-kappab can be inhibited by novel pharmacological agents in osteoarthritis. Rheumatology (Oxford) 2007, 46:752-758.

37. Marcu KB, Otero M, Olivotto E, Borzi RM, Goldring MB: NF-kappaB signaling: multiple angles to target OA. Curr Drug Targets 2010, 11:599-613.

38. Simmonds RE, Foxwell BM: Signalling, inflammation and arthritis: NF-kappaB and its relevance to arthritis and inflammation. Rheumatology (Oxford) 2008, 47:584-590.

39. Szliszka E, Czuba ZP, Mertas A, Paradysz A, Krol W: The dietary isoflavone biochanin-A sensitizes prostate cancer cells to TRAlL-induced apoptosis. Urol Oncol 2013, 31(3):331-342.

40. Setton LA, Elliott DM, Mow VC: Altered mechanics of cartilage with osteoarthritis: human osteoarthritis and an experimental model of joint degeneration. Osteoarthritis Cartilage 1999, 7:2-14.

41. Laverty S, Girard CA, Williams JM, Hunziker EB, Pritzker KP: The OARSI histopathology initiative - recommendations for histological assessments of osteoarthritis in the rabbit. Osteoarthritis Cartilage 2010, 18(Suppl 3):S53-S65.

42. Pickarski M, Hayami T, Zhuo Y, Duong le T: Molecular changes in articular cartilage and subchondral bone in the rat anterior cruciate ligament transection and meniscectomized models of osteoarthritis. BMC Musculoskelet Disord 2011, 12:197.

43. Li X, Li J, Cheng K, Lin Q, Wang D, Zhang H, An H, Gao M, Chen A: Effect of low-intensity pulsed ultrasound on MMP-13 and MAPKs signaling pathway in rabbit knee osteoarthritis. Cell Biochem Biophys 2011, 61:427-434.

doi:10.1186/1472-6882-14-444

Cite this article as: Wu et al:: Protective effects of biochanin A on articular cartilage: in vitro and in vivo studies. BMC Complementary and Alternative Medicine 2014 14:444.

\section{Submit your next manuscript to BioMed Central and take full advantage of:}

- Convenient online submission

- Thorough peer review

- No space constraints or color figure charges

- Immediate publication on acceptance

- Inclusion in PubMed, CAS, Scopus and Google Scholar

- Research which is freely available for redistribution 